TRANSACTIONS OF THE

AMERICAN MATHEMATICAL SOCIETY

Volume 348, Number 3, March 1996

\title{
AFFINE DUPIN SURFACES
}

\author{
ROSS NIEBERGALL AND PATRICK J. RYAN
}

\begin{abstract}
In this paper we study nondegenerate affine surfaces in $\mathbb{R}^{3}$ whose affine principal curvatures are constant along their lines of curvature. We give a complete local classification of these surfaces assuming that the lines of curvature are planar, and there are no umbilics.
\end{abstract}

\section{INTRODUCTION}

The cyclides of Dupin were characterized classically by the property that both sheets of the focal set are curves. This observation is a starting point for the theory of Dupin submanifolds, which has developed over the past 15 years, see for example $[\mathrm{CR}, \mathrm{C}]$.

In this paper, we initiate the study of the Dupin condition in affine differential geometry, beginning with the case of surfaces. Although many affine surfaces have been studied over the past decade, most examples have constant principal curvatures, i.e. they are isoparametric in the sense of [NR1]. The new examples characterized in this paper have one nonconstant and one constant principal curvature.

Although the Dupin condition in the Riemannian case implies that the lines of curvature are planar-in fact they are circles or lines, it is still an open question whether or not this holds for affine Dupin surfaces. Here, we obtain a local classification of affine Dupin surfaces in 3-space whose lines of curvature are planar.

After some preliminary background in $\S 1$, we show in $\S 2$ that it is impossible for both principal curvatures of an affine Dupin surface with planar lines of curvature to be non-constant. In $\S 3$ we state Vrancken's result [V] for the case where both principal curvatures are constant. The general form for the case of precisely one constant principal curvature is derived in $\S 4$. The possibilities naturally divide into three cases which are treated in $\S 5, \S 6$, and $\S 7$ respectively. For convenience in stating the main theorem, we define a point of an affine Dupin surface to be of

Type 0 if it has a neighborhood that is isoparametric;

Type 1 if it has a neighborhood satisfying Case 1 of $\S 4$;

Type 2 if it has a neighborhood satisfying Case 2 of $\S 4$;

Type 3 if it has a neighborhood satisfying Case 3 of $\S 4$.

We will prove the following local classification (see Theorem 7).

Received by the editors September 1, 1994 and, in revised form, February 6, 1995

1991 Mathematics Subject Classification. Primary 53A15; Secondary 53A05, 53B25.

Research supported by an NSERC Postdoctoral Fellowship and NSERC Operating Grant OGP0002501. 
Main Theorem. Let $f: M \rightarrow \mathbb{R}^{3}$ be an affine Dupin surface with (distinct real) principal curvatures $\lambda$ and $\mu$ and planar lines of curvature. Then there is a dense set of points of $M$, each of which is of one of the types 0-3.

For a precise description of each case, see the statements of Theorems 2, 4, 5, and 6 .

The authors wish to thank the referee for suggesting simplifications of the proofs of Lemmas 2.1 and 2.3 .

\section{Affine surfaces}

Let $f: M^{2} \rightarrow \mathbb{R}^{3}$ be a nondegenerate affine surface. For the early calculations we will be brief, since similar things can be found in any paper on affine surfaces, for example $[\mathrm{MR}]$. Assume that the shape operator is diagonalizable with distinct real principal curvatures. We work locally. In other words, we work in a simply connected open set $U$ with a choice of affine normal $\xi$. Then we have smooth principal curvature functions $\lambda$ and $\mu$ and can choose (uniquely up to a sign) corresponding principal vector fields $\{X, Y\}$ which are orthonormal with respect to the affine metric $h$. This means that $S X=\lambda X, S Y=\mu Y$, $h(X, X)=\varepsilon, h(X, Y)=0, h(Y, Y)=1$, where $\varepsilon= \pm 1$ and $S$ is the shape operator.

We now express the induced connection $\nabla$ in terms of this basis. Specifically, there are real-valued functions $a, b, c, e, \gamma$, and $\sigma$ such that

$$
\begin{aligned}
& \nabla_{X} X=a X+c Y, \\
& \nabla_{X} Y=\gamma X-a Y, \\
& \nabla_{Y} X=-b X+\sigma Y, \\
& \nabla_{Y} Y=e X+b Y,
\end{aligned}
$$

where the apolarity condition has been taken into account. Furthermore, we can express $c$ and $e$ in terms of the other parameters by making use of the Codazzi equation for $h$ which yields

$$
\varepsilon \gamma+c=-2 \varepsilon b, \quad \sigma+\varepsilon e=-2 a .
$$

We also note that the Codazzi equation for $S$ gives

$$
\gamma=\frac{Y \lambda}{\mu-\lambda}, \quad \sigma=\frac{X \mu}{\lambda-\mu}
$$

and we will use this in the proof of Lemma 2.1.

The familiar relationships among the usual flat connection $D$ on $\mathbb{R}^{3}$, the induced connection $\nabla$, the affine metric $h$, the affine normal $\xi$ and the principal curvatures $\lambda, \mu$, translate into

$$
\begin{aligned}
D_{X} X & =a X-\varepsilon(2 b+\gamma) Y+\varepsilon \xi, \\
D_{X} Y & =\gamma X-a Y, \\
D_{Y} X & =-b X+\sigma Y, \\
D_{Y} Y & =-\varepsilon(2 a+\sigma) X+b Y+\xi, \\
D_{X} \xi & =-\lambda X \\
D_{Y} \xi & =-\mu Y .
\end{aligned}
$$




\section{The Dupin CONDITION}

A nondegenerate affine surface with real distinct principal curvatures will be called an affine Dupin surface if, in the notation of the preceding section, $X \lambda=$ $Y \mu=0$. This definition is consistent with the characterization in terms of focal sets given in Proposition $\mathrm{C}$ of [NR2]. In other words, the focal set consists of curves. The Dupin condition implies relationships among the functions $a, b, \gamma, \sigma$ which we now explore.

Lemma 2.1. For an affine Dupin surface, $Y \sigma+b \sigma=0$ and $X \gamma+a \gamma=0$.

Proof. Since the surface is Dupin, $Y \mu=0$. Thus from (1.3) and (1.2) we have

$$
\left(D_{X} Y-D_{Y} X\right) \mu=(\gamma+b) X \mu=\sigma(\gamma+b)(\lambda-\mu) .
$$

On the other hand,

$$
[X, Y] \mu=X Y \mu-Y X \mu=-Y X \mu
$$

But

$$
Y X \mu=(Y \sigma)(\lambda-\mu)+\sigma(Y \lambda)=(Y \sigma-\sigma \gamma)(\lambda-\mu)
$$

Noting that $\lambda \neq \mu$ and comparing (2.1) with (2.2), we see that

$$
Y \sigma=-(\gamma+b) \sigma+\gamma \sigma=-b \sigma
$$

The fact that

$$
X \gamma=-a \gamma
$$

can be verified in a similar fashion.

Lemma 2.2. For an affine Dupin surface, the Gauss equation results in

$$
\begin{gathered}
X b+Y a+6 a b+3 a \gamma+3 b \sigma=0, \\
X \sigma+\varepsilon Y(2 b+\gamma)+6 \varepsilon b^{2}+5 \varepsilon b \gamma-a \sigma+\varepsilon \gamma^{2}+\sigma^{2}=-\varepsilon \mu, \\
Y \gamma+\varepsilon X(2 a+\sigma)+6 \varepsilon a^{2}+5 \varepsilon a \sigma-b \gamma+\varepsilon \sigma^{2}+\gamma^{2}=-\lambda .
\end{gathered}
$$

Proof. Substitute into the Gauss equations for $R(X, Y) X$ and $R(X, Y) Y$.

As mentioned in the introduction, we still do not know whether the lines of curvature for an affine Dupin surface must be planar. However, this property is necessary for our classification proof.

We take our first step towards this classification by showing that for an affine Dupin surface with planar lines of curvature, at least one of the principal curvatures is typically constant. For the precise statement, see Theorem 1. 
Lemma 2.3. For an affine Dupin surface with planar lines of curvature, we have (in the notation introduced above)

$$
Y a=(a+\sigma) b,
$$

$$
X b=(b+\gamma) a,
$$

and the first Gauss equation simplifies to

$$
2 a b+b \sigma+a \gamma=0
$$

Proof. Since the line of curvature associated with the principal direction $X$ is planar, we can conclude that $X, D_{X} X$, and $D_{X}\left(a X-D_{X} X\right)$, are linearly dependent. But this latter vector is

$$
\begin{aligned}
D_{X}(\varepsilon(2 b+\gamma) Y-\varepsilon \xi) & =\varepsilon(X(2 b+\gamma) Y+(2 b+\gamma)(\gamma X-a Y)+\lambda X) \\
& =\varepsilon(X(2 b+\gamma)-a(2 b+\gamma)) Y+\varepsilon((2 b+\gamma) \gamma+\lambda) X .
\end{aligned}
$$

Linear dependence immediately implies that the coefficient of $Y$ is zero, that is

$$
X(2 b+\gamma)=a(2 b+\gamma)
$$

which can be simplified using equation (2.4) to get

$$
X b=a(b+\gamma) .
$$

The other equations follow similarly by considering $D_{Y} Y$.

We now prove our local result about the constancy of one principal curvature.

Theorem 1. For an affine Dupin surface with planar lines of curvature, let $p_{0}$ be a point where a principal curvature $\mu$ satisfies $d \mu \neq 0$. Then the other principal curvature $\lambda$ must be constant in a neighborhood of $p_{0}$.

Proof. Suppose that $Y \lambda \neq 0$ and $X \mu \neq 0$ at some point. Then in some open neighborhood of this point, $\gamma$ and $\sigma$ must be nonzero by (1.2). From (2.10) we know that the first Gauss equation is

$$
2 a b+b \sigma+a \gamma=0
$$

and

$$
\begin{aligned}
& Y a=(a+\sigma) b, \\
& X b=(b+\gamma) a .
\end{aligned}
$$

Differentiating (2.10), we get

$$
(2 b+\gamma) X a+b X \sigma+a \sigma \gamma=0,
$$

$$
(2 a+\sigma) Y b+a Y \gamma+b \sigma \gamma=0
$$


Notice that if either $a$ or $b$ vanishes, then so does the other. This is evident from (2.10). It is also clear from (2.10) that if $a$ and $b$ are nonzero, then $2 a+\sigma \neq 0$ and $2 b+\gamma \neq 0$.

We first consider the possibility that $a$ and $b$ are nonzero. We then solve (2.11) for $X \sigma$, and (2.12) for $Y \gamma$ obtaining

$$
\begin{aligned}
& X \sigma=-\frac{(2 b+\gamma) X a+a \sigma \gamma}{b}, \\
& Y \gamma=-\frac{(2 a+\sigma) Y b+b \gamma \sigma}{a} .
\end{aligned}
$$

We can then insert these values into (2.6) and (2.7), to get

$$
\begin{aligned}
& -(2 a b+a \gamma) X a-\varepsilon b^{2} \gamma \sigma+6 \varepsilon b^{3} a-a^{2} \gamma \sigma-\varepsilon b \sigma Y b \\
& +\varepsilon \gamma^{2} a b+\sigma^{2} a b+5 \varepsilon b^{2} \gamma a-a^{2} \sigma b+\varepsilon \mu a b=0, \\
& -(2 a b+b \sigma) Y b-\varepsilon a^{2} \gamma \sigma+6 \varepsilon a^{3} b-b^{2} \gamma \sigma-\varepsilon a \gamma X a \\
& +\varepsilon \sigma^{2} a b+\gamma^{2} a b+5 \varepsilon a^{2} \sigma b-b^{2} \gamma a+\lambda a b=0 .
\end{aligned}
$$

Now, by multiplying $-(2 b a+b \sigma)$ times (2.13) and adding it to $\varepsilon b \sigma$ times (2.14), we get an expression with neither $X a$ nor $Y b$, since the coefficient is zero by (2.10).

$$
\begin{aligned}
& 2 \varepsilon a^{2} \gamma \sigma-12 b^{3} a+8 \varepsilon a^{2} \sigma b+4 \varepsilon \sigma^{2} a b-10 b^{2} \gamma a \\
& \quad-2 \gamma^{2} a b-2 \mu a b-4 b^{2} \gamma \sigma-6 \sigma b^{3}-\sigma \mu b+b \sigma \lambda=0 .
\end{aligned}
$$

This can then be reduced using (2.10) to get

$$
2 \sigma \gamma\left(\varepsilon a^{2}+b^{2}\right)=a \gamma \mu+b \sigma \lambda
$$

Differentiating equation (2.15) first with respect to $X$ and then with respect to $Y$, we get

$$
\begin{aligned}
& \left(-2 \varepsilon \gamma^{2} a^{2}-2 \gamma^{2} b^{2}-4 \varepsilon \gamma b a^{2}-4 \gamma b^{3}+4 \varepsilon \gamma \sigma a b-\gamma \mu b+2 \lambda b^{2}+\lambda b \gamma\right) X a \\
& \quad-2 \varepsilon a^{3} \gamma \sigma b+2 a \gamma \sigma b^{3}-2 \varepsilon a^{3} g^{2} \sigma \\
& \quad+2 a \gamma^{2} \sigma b^{2}+a \gamma b \sigma \mu-a \sigma \lambda b^{2}+a^{2} \gamma b \mu-a \gamma b \sigma \lambda=0, \\
& \left(-4 a \sigma b^{2}-4 \varepsilon a^{3} \sigma+4 a \gamma \sigma b+2 a^{2} \mu+a \mu \sigma-\sigma \lambda a-2 \varepsilon \sigma^{2} a^{2}-2 \sigma^{2} b^{2}\right) Y b \\
& \quad+2 \varepsilon b \gamma \sigma^{2} a^{2}-2 b^{3} \gamma \sigma^{2}+a \sigma \lambda b^{2}+a \gamma b \sigma \lambda+2 \varepsilon a^{3} \gamma \sigma b \\
& \quad-2 a \gamma \sigma b^{3}-a^{2} \gamma b \mu-a \gamma b \sigma \mu=0 .
\end{aligned}
$$

Now, from equation (2.10) 


$$
\gamma=-\frac{2 a b+b \sigma}{a}
$$

We can then solve (2.13) for $\mu$ and (2.14) for $\lambda$, to get

$$
\begin{aligned}
& \lambda=-\frac{(2 a+\sigma)\left(3 \varepsilon a^{3}+2 \varepsilon \sigma a^{2}-Y b a+\varepsilon X a a+3 b^{2} a+2 b^{2} \sigma\right)}{a^{2}}, \\
& \mu=-\frac{\varepsilon \sigma\left(X a a+a^{3}+2 \sigma a^{2}-\varepsilon Y b a+\varepsilon b^{2} a+2 \varepsilon b^{2} \sigma\right)}{a^{2}} .
\end{aligned}
$$

Using (2.18) and (2.19), we can then simplify (2.16) and (2.17), to get

$$
\begin{gathered}
-4 \varepsilon b^{2} \sigma\left(2 X a a+X a \sigma+2 a^{3}+3 \sigma a^{2}+\sigma^{2} a\right)=0, \\
\frac{4 b^{2} \sigma\left(2 b^{2} a^{2}-\sigma Y b a-2 Y b a^{2}+3 b^{2} \sigma a+b^{2} \sigma^{2}\right)}{a}=0 .
\end{gathered}
$$

This will now allow us to solve for $X a$ and $Y b$. In particular, since $2 a+\sigma \neq 0$, we have

$$
\begin{aligned}
& X a=-a(a+\sigma), \\
& Y b=\frac{b^{2}}{a}(a+\sigma) .
\end{aligned}
$$

Reinserting these values into (2.19) results in a further simplification and yields

$$
\mu=-\frac{\sigma^{2}\left(\varepsilon a^{2}+b^{2}\right)}{a^{2}} .
$$

Differentiating this in the $Y$ direction, we have

$$
Y \mu=\frac{2 \sigma^{2} b\left(\varepsilon a^{2}+b^{2}\right)}{a^{2}} .
$$

Hence, $Y \mu=-2 b \mu$. This must be zero, since the surface is Dupin. But this says that $\mu$ is identically zero, a contradiction.

The other possibility is that both $a$ and $b$ are zero. Then the Gauss equations (2.6) and (2.7) would simplify to

$$
\begin{aligned}
& X \sigma+\varepsilon Y \gamma+\varepsilon \gamma^{2}+\sigma^{2}=-\varepsilon \mu \\
& Y \gamma+\varepsilon X \sigma+\varepsilon \sigma^{2}+\gamma^{2}=-\lambda
\end{aligned}
$$

Multiplying (2.20) by $\varepsilon$ yields $\lambda=\mu$, contradicting the assumption that the principal curvatures are distinct. 


\section{THE ISOPARAMETRIC CASE}

The following is a result of Vrancken [V] translated into our formalism.

Theorem 2. Let $f: M \rightarrow \mathbb{R}^{3}$ be an affine Dupin surface with (distinct real) constant principal curvatures $\lambda$ and $\mu$. Then for any point $p_{0}$ in $M$ there is a coordinate system $\{u, v\}$ valid near $p_{0}$ and constant vectors $K, L, N$, and $C$ such that in the domain of these coordinates,

$$
f(u, v)=u L+v N+\left(\frac{1}{2} u^{2}+c v G(v)\right) K+C
$$

where $G^{\prime}(v)=v^{-2}\left(\alpha^{2} \pm v^{2}\right)^{-1 / 3}$ and $c$ and $\alpha$ are constants. Also, one principal curvature must be zero.

Note that this includes the familiar homogeneous affine surfaces [NS] which can be expressed as $\left(U^{2}-W\right)^{3} V^{2}=1$ in a suitable affine coordinate system. These are obtained by setting $\alpha=0$.

We now consider the possibility of nonconstant principal curvatures.

\section{Affine Dupin surfaces with one nonconstant principal CuRvature AND PLANAR LINES OF CURVATURE}

In this section consider an affine Dupin surface with planar lines of curvature that is not isoparametric. From the proof of Theorem 1, we must have $(Y \lambda)(X \mu)=0$. Hence, there is some open subset of $U$ on which one of the principal curvatures is constant. Without loss of generality, we will assume that $\lambda$ is constant, so that $X \lambda=Y \lambda=0$, while $X \mu \neq 0$. Then $\gamma=0$ but $\sigma \neq 0$.

Since $\gamma=0$, the Gauss equation (2.10) becomes

$$
b(2 a+\sigma)=0 .
$$

At any particular point, there are three distinct possibilities, which can be expressed using (1.1) as follows:

Case 1. $b=0$ and $2 a+\sigma=0$, i.e. $\nabla_{Y} Y=0$.

Case 2. $b \neq 0$ and $2 a+\sigma=0$, i.e. $\nabla_{Y} Y$ is a nonzero multiple of $Y$.

Case 3. $b=0$ and $2 a+\sigma \neq 0$, i.e. $\nabla_{Y} Y$ is a nonzero multiple of $X$.

Note that the cases are distinguished by the behavior of the principal vector corresponding to the nonconstant principal curvature.

If either Case 2 or Case 3 holds at a particular point, then it holds in a neighborhood of the point. The same cannot be said for Case 1. However we will first examine the consequences of each of these conditions being satisfied locally. Later, we will see how the solutions fit together.

Before this, we will perform some computations which are applicable to all three cases. In particular, we need to establish a system of principal coordinates. The following is a standard result of surface theory; see, for example $[\mathrm{K}]$, p. 51.

Lemma 4.1. Let $X$ and $Y$ be linearly independent vector fields on a 2-dimensional manifold $M$. In a neighborhood of any point $p_{0} \in M$ there exist positive functions $\zeta$ and $\tau$ such that $\bar{X}=\zeta X$ and $\bar{Y}=\tau Y$ satisfy $[\bar{X}, \bar{Y}]=0$, and local coordinates $\{x, y\}$ such that $\bar{X}=\frac{\partial}{\partial x}$ and $\bar{Y}=\frac{\partial}{\partial y}$.

In the case of an affine Dupin surface we can be more explicit about $\zeta$ and $\tau$. 
Lemma 4.2. For an affine Dupin surface satisfying the hypotheses of Theorem 1 , the functions $\zeta$ and $\tau$ guaranteed by Lemma 4.1 must satisfy $Y(\ln \zeta)=b$, and $X(\ln \tau)=a+\sigma$, respectively. Conversely, any two positive functions $\zeta$ and $\tau$ satisfying these conditions give rise to a local coordinate system as in Lemma 4.1.

Proof. First note that $\sigma \neq 0$, since $X \mu \neq 0$ by (1.2). Let $X$ and $Y$ be the principal vectors used in Theorem 1 and let $\bar{X}=\zeta X$ and $\bar{Y}=\tau Y$. Then

$$
D_{\bar{X}} \bar{Y}=\zeta((X \tau) Y+\tau(-a Y))
$$

and

$$
D_{\bar{Y}} \bar{X}=\tau((Y \zeta) X+\zeta(-b X+\sigma Y)),
$$

where we have used Lemma 2.1, (1.3) and the fact that $\gamma=0$. Thus

$$
[\bar{X}, \bar{Y}]=\zeta((X \tau)-\tau(a+\sigma)) Y-\tau(Y \zeta-b \zeta) X
$$

This shows that the differential equations for $\zeta$ and $\tau$ are equivalent to the vanishing of $[\bar{X}, \bar{Y}]$ and hence to the existence of the desired coordinate system.

It is easy to check that $\zeta$ may be taken to be $\frac{1}{\sigma}$ (Lemma 2.1) and we shall do that from now on. For the moment, we will continue working in terms of a general $\tau$ and make a different choice for each of the three cases. Since we will need some flexibility in our choice of $\tau$, we establish the following technical lemma.

Lemma 4.3. Suppose that a coordinate system $\{x, y\}$ is given as in Lemma 4.2 using $\zeta=\frac{1}{\sigma}$ and some $\tau$. Let $\tilde{\tau}$ be a positive function satisfying $X(\ln \tilde{\tau})=a+\sigma$. Then there is a function $\tilde{y}$ such that $\{x, \tilde{y}\}$ is a coordinate system satisfying $\frac{\partial}{\partial \tilde{y}}=$ $\tilde{\tau} Y$.

Proof. Use Lemma 4.1 to find a coordinate system $\{\tilde{x}, \tilde{y}\}$ using $\tilde{\zeta}=\frac{1}{\sigma}$ and the given $\tilde{\tau}$. Write down the transformation equations for the coordinate vector fields and observe that $x$ and $\tilde{x}$ must differ by a constant. Thus $\tilde{x}$ may be replaced by $x$ in the second coordinate system.

The purpose of Lemma 4.3 is to allow us to use the coordinate $x$ in our definition of $\tau$ in Theorems 4 and 6 .

Let us now rewrite (1.3) in terms of $\bar{X}$ and $\bar{Y}$.

$$
\begin{gathered}
D_{\bar{X}} \bar{Y}=\zeta \tau \sigma Y=\bar{Y} \\
D_{\bar{Y}} \bar{X}=\tau \zeta \sigma Y=\bar{Y} \\
D_{\bar{X}} \bar{X}=\frac{a-\sigma_{x}}{\sigma} \bar{X}-2 \varepsilon \frac{b}{\tau \sigma^{2}} \bar{Y}+\frac{\varepsilon}{\sigma^{2}} \xi \\
D_{\bar{Y}} \bar{Y}=-\varepsilon \tau^{2} \sigma(2 a+\sigma) \bar{X}+\left(\frac{\tau_{y}}{\tau}+b \tau\right) \bar{Y}+\tau^{2} \xi \\
D_{\bar{X}} \xi=-\lambda \bar{X} \\
D_{\bar{Y}} \xi=-\mu \bar{Y} .
\end{gathered}
$$


We now describe a system of differential equations satisfied by the immersing function $f$. Note that $D_{\bar{X}} \bar{Y}$ can be replaced by $f_{x y}, \bar{X}$ by $f_{x}$ and so on, to yield the following version of (1.3) valid in the coordinate neighborhood.

$$
\begin{gathered}
f_{x y}=f_{y}, \\
f_{x x}=\frac{a-\sigma_{x}}{\sigma} f_{x}-2 \varepsilon \frac{b}{\tau \sigma^{2}} f_{y}+\frac{\varepsilon}{\sigma^{2}} \xi \\
f_{y y}=-\varepsilon \tau^{2} \sigma(2 a+\sigma) f_{x}+\left(\frac{\tau_{y}}{\tau}+b \tau\right) f_{y}+\tau^{2} \xi, \\
\xi_{x}=-\lambda f_{x}, \\
\xi_{y}=-\mu f_{y} .
\end{gathered}
$$

We also get from (1.2) a differential equation for $\mu$,

$$
\mu_{x}=\frac{1}{\sigma} X \mu=\lambda-\mu(x)
$$

Since $\lambda$ is constant, and $\mu$ is a function of $x$ alone, there is a constant $c$ such that

$$
\mu(x)=\lambda+c e^{-x} .
$$

Now, (4.2) can be solved to show that

$$
f=e^{x} g(y)+h(x)
$$

where $g(y)$ and $h(x)$ are vector-valued functions, and (4.5-4.6) can be solved to show that

$$
\begin{aligned}
\xi & =-\left(\lambda e^{x}+c\right) g(y)-\lambda h(x)+V \\
& =-\mu e^{x} g(y)-\lambda h(x)+V
\end{aligned}
$$

for some constant vector $V$.

Remark. Before proceeding with our classification, it is appropriate to make a few observations about the domains of definition of the various parameters introduced and their uniqueness. We start with a simply connected region $U$ of $M$. If the affine metric is definite, we choose $\xi$ so that $h$ is positive definite. If $h$ is indefinite, either choice of $\xi$ is equally good. The condition $(X \lambda)(Y \mu)=0$ is independent of choice of which principal curvature is called $\lambda$ and which is called $\mu$. For a Dupin surface with planar lines of curvature, this condition is true by Theorem 1. Disregarding the isoparametric case for the moment, we know that there is an open subset $U_{1} \subseteq U$ on which one principal curvature is constant and the other has nonvanishing differential. If $h$ is definite, we obviously lose no generality in calling the constant principal curvature $\lambda$. In the indefinite case, the same can be achieved 
by making the correct choice of affine normal. Now that $\xi, \lambda$, and $\mu$ have been chosen, we note that $X$ and $Y$ are unique only up to a sign. So are the functions defined in (1.3). However, the condition $b(2 a+\sigma)=0$ is independent of choice of $X$ and $Y$, as are the three pointwise conditions into which it is decomposed.

For any point $p_{0}$ in $U_{1}$ there is a coordinate neighborhood $U_{2} \subseteq U_{1}$ with coordinates $\{x, y\}$ as described above. Further, we arrange that the coordinates of $p_{0}$ are $(0,0)$ in this system. Once this coordinate system has been fixed, the constants and functions occurring in (4.7), (4.8), and (4.9) are determined. Our classification will amount to determining the possible values for these constants and functions and arranging the resulting surfaces in some intelligible list.

In coming up with specific immersing functions in our subsequent work, it is useful to recall that the definition of affine normal requires that the area form $\nu$ determined by the affine metric must coincide with the induced area form $\theta$ determined by $\xi$. This fact is particularly important in $\S 5$ and $\S 6$.

Although the following theorem does not include all the detail necessary for our classification proof, it gives a good picture of our progress to this point.

Theorem 3. Let $f: M \rightarrow \mathbb{R}^{3}$ be an affine Dupin surface with (distinct real) principal curvatures $\lambda$ and $\mu$ and planar lines of curvature. Let $p_{0}$ be a point of $M$ where $d \mu \neq 0$. Then there is a coordinate system $\{x, y\}$ with origin $p_{0}$ such that in the domain of these coordinates,

1. $\lambda$ is constant;

2. $\mu=\lambda+c e^{-x}$ for some constant $c$;

3. $f(x, y)=e^{x} g(y)+h(x)$ for some vector-valued functions $g$ and $h$;

4. $\xi=-\left(\lambda e^{x}+c\right) g(y)-\lambda h(x)+V$ for some constant vector $V$.

$$
\text { 5. CASE 1: } b=0 \text { AND } 2 a+\sigma=0 . \nabla_{Y} Y=0
$$

Theorem 4. Let $f: M \rightarrow \mathbb{R}^{3}$ be an affine Dupin surface with exactly one nonconstant principal curvature $\mu$ and planar lines of curvature. Let $X$ and $Y$ be orthonormal (with respect to $h$ ) principal vectors corresponding to principal curvatures $\lambda$ and $\mu$ respectively. Suppose that $\nabla_{Y} Y=0$. Let $p_{0}$ be a point of $M$ where $d \mu \neq 0$. Then there is an affine coordinate system $\{U, V, W\}$ for $\mathbb{R}^{3}$ such that $f$ maps a neighborhood of $p_{0}$ onto an open subset of one of the following surfaces

$$
\begin{array}{r}
U^{2} \pm V^{2}=e^{2 W}, \\
U^{2} \pm V^{2}=\sin ^{2} W, \\
U^{2} \pm V^{2}=\sinh ^{2} W \\
U^{2} \pm V^{2}=\cosh ^{2} W
\end{array}
$$

Further, the constant principal curvature $\lambda$ is zero.

Proof. We assume that we have a surface as described in Theorem 3 satisfying the condition $\nabla_{Y} Y=0$. The condition $(X \tau) / \tau=a+\sigma$ is expressed in local coordinates as

$$
\frac{\tau_{x}}{\tau}=\frac{a+\sigma}{\sigma},
$$

which reduces to

$$
\frac{\tau_{x}}{\tau}=\frac{1}{2},
$$


since $\sigma=-2 a$. One solution to this equation is $\tau=e^{x / 2}$ and we can use Lemma 4.3 to redefine the coordinate system so that this expression for $\tau$ applies.

The second Gauss equation (2.6) becomes

$$
4 a a_{x}+6 a^{2}=-\varepsilon\left(\lambda+c e^{-x}\right),
$$

and the third (2.7) becomes

$$
\lambda=0
$$

Then (5.2) can be solved for $a$ to get

$$
a^{2}=-\frac{1}{4} \varepsilon c e^{-x}+e^{-3 x} c_{1}
$$

for some constant $c_{1}$. Also (4.4) becomes

$$
g_{y y}=-c g(y)+V,
$$

which can be solved to get

$$
\begin{aligned}
& g(y)=\cosh (\omega y) A+\sinh (\omega y) B+\frac{1}{c} V \quad \text { or } \\
& g(y)=\cos (\omega y) A+\sin (\omega y) B+\frac{1}{c} V .
\end{aligned}
$$

where $\omega=\sqrt{|c|}$. Lastly, (4.3) may be simplified using (5.2) to

$$
h_{x x}=-\left(\frac{a_{x}}{a}+\frac{1}{2}\right) h_{x}+\frac{\varepsilon}{4 a^{2}} V=\frac{4 c_{1}}{4 c_{1}-\varepsilon c e^{2 x}} h_{x}+\frac{\varepsilon}{-\varepsilon c e^{-x}+4 e^{-3 x} c_{1}} V .
$$

This equation can be solved for $h_{x}$ to get a constant vector $C$ such that

$$
h_{x}=\frac{e^{x}}{\left(4 c_{1}-\varepsilon c e^{2 x}\right)^{1 / 2}} C-\frac{e^{x}}{c} V .
$$

There are four different forms that $h$ can take, depending on the values of $\varepsilon, c$, and $c_{1}$. Note first that $4 c_{1}-\varepsilon c$ must be positive since the solution must exist near $(0,0)$. Write $\alpha=2 \sqrt{\left|c_{1}\right|}$. If $c_{1}>0$ and $\varepsilon c<0$ the solution is

$$
h(x)=\frac{1}{\omega} \operatorname{arcsinh}\left(\frac{\omega e^{x}}{\alpha}\right) C-\frac{e^{x}}{c} V+E,
$$

and if $c_{1}>0$ and $\varepsilon c>0$ the solution is

$$
h(x)=\frac{1}{\omega} \arcsin \left(\frac{\omega e^{x}}{\alpha}\right) C-\frac{e^{x}}{c} V+E .
$$

If $c_{1}<0$ and $\varepsilon c<0$, then

$$
h(x)=\frac{1}{\omega} \operatorname{arccosh}\left(\frac{\omega e^{x}}{\alpha}\right) C-\frac{e^{x}}{c} V+E .
$$


Lastly, if $c_{1}=0$ and hence $\varepsilon c<0$,

$$
h(x)=\frac{x}{\omega} C-\frac{e^{x}}{c} V+E .
$$

In all cases, $E$ is a constant vector. Now, let $k(x)$ be the coefficient of $C$ in equations (5.5-5.7). Then the immersing function $f(x, y)$ takes on the form

$$
f(x, y)=e^{x} \cosh (\omega y) A+e^{x} \sinh (\omega y) B+k(x) C+E,
$$

or a similar expression using trigonometric functions (see 5.4). The calculation of $\theta\left(f_{x}, f_{y}\right)$ is easy in this case and yields

$$
\theta\left(f_{x}, f_{y}\right)=\frac{c \omega e^{2 x}}{\sqrt{4 c_{1}-\varepsilon c e^{2 x}}} \operatorname{det}(A, B, C),
$$

while

$$
\nu\left(f_{x}, f_{y}\right)= \pm \frac{\tau}{\sigma}= \pm \frac{e^{2 x}}{\sqrt{4 c_{1}-\varepsilon c e^{2 x}}} .
$$

Therefore $A, B$, and $C$ must be linearly independent and, in particular,

$$
\operatorname{det}(A, B, C)= \pm \frac{1}{\omega c} .
$$

We now show that the surfaces we have been discussing have simple equations with respect to a convenient affine coordinate system for 3-space. For example, (5.9) may be rewritten

$$
\begin{aligned}
f(x, y)= & \frac{\omega}{\alpha} e^{x} \cosh (\omega y)\left(\frac{\alpha}{\omega} A\right)+\frac{\omega}{\alpha} e^{x} \sinh (\omega y)\left(\frac{\alpha}{\omega} B\right) \\
& +\omega k(x)\left(\frac{1}{\omega} C\right)+E .
\end{aligned}
$$

If we use $E$ as origin and the indicated multiples of $A, B$, and $C$ as the basis for coordinates $U, V$, and $W$, then

$$
W=\operatorname{arcsinh}\left(\frac{\omega e^{x}}{\alpha}\right),
$$

so that

$$
U^{2}-V^{2}=\sinh ^{2} W
$$

Similarly, we can express (5.6) and (5.7) by

$$
\begin{gathered}
U^{2}-V^{2}=\sin ^{2} W \\
U^{2}-V^{2}=\cosh ^{2} W .
\end{gathered}
$$

If $c_{1}=0$, the basis must be modified as indicated by the following form of $(5.9)$

$$
f(x, y)=e^{x} \cosh (\omega y) A+e^{x} \sinh (\omega y) B+x\left(\frac{1}{\omega} C\right) .
$$


This leads to the equation

$$
U^{2}-V^{2}=e^{2 W}
$$

When $c<0$, the solution for $g(y)$ involves trigonometric rather than hyperbolic functions, and the algebraic equations of the resulting surfaces are

$$
\begin{aligned}
U^{2}+V^{2} & =\sinh ^{2} W, \\
U^{2}+V^{2} & =\sin ^{2} W, \\
U^{2}+V^{2} & =\cosh ^{2} W, \\
U^{2}+V^{2} & =e^{2 W} .
\end{aligned}
$$

Remark. For these examples, one can check that planes of the form $W=c$ cut the surface in lines of curvature that are ellipses or hyperbolas whose centers lie along the $W$-axis. These centers are the $\mu$-focal points. The other lines of curvature are the profile curves cut out by planes through the $W$-axis. They correspond to the principal curvature $\lambda=0$, and the $\lambda$-focal set is empty.

\section{CASE 2: $b \neq 0$ AND $2 a+\sigma=0 . \nabla_{Y} Y$ IS A Multiple of $Y$}

In this section, we assume that we have a surface as described in Theorem 3 satisfying the condition in the heading of Case 2. Our result is the following.

Theorem 5. Let $f: M \rightarrow \mathbb{R}^{3}$ be an affine Dupin surface with exactly one nonconstant principal curvature $\mu$ and planar lines of curvature. Let $X$ and $Y$ be orthonormal (with respect to $h$ ) principal vectors corresponding to principal curvatures $\lambda$ and $\mu$ respectively. Suppose that $\nabla_{Y} Y$ is a nonzero multiple of $Y$. Let $p_{0}$ be a point of $M$ where $d \mu \neq 0$. Then there is a coordinate system $\{x, y\}$ with origin $p_{0}$ and real constants $c_{1}, c_{2}, c$, and $k$, such that, in the domain of these coordinates, if we write

$$
\begin{gathered}
\varphi(y)=\left(12 c_{2}-3 \varepsilon k e^{4 y}-2 c e^{6 y}\right)^{1 / 2} \\
\Gamma(y)=\int_{0}^{y} \frac{e^{6 t}}{\varphi(t)^{3}} d t, \quad \Lambda(y)=\int_{0}^{y} \frac{e^{4 t}}{\varphi(t)^{3}} d t \\
\Psi(x)=\int_{0}^{x} \frac{e^{t}}{\left(4 c_{1}+k e^{2 t}\right)^{1 / 2}} d t
\end{gathered}
$$

either

$$
\begin{aligned}
f(x, y)= & 6 \varphi(y) e^{x} A+\Psi(x) B+\left(6 \varepsilon \varphi(y) e^{x} \Lambda(y)-\frac{e^{x}}{k}\right) T \\
& +6 \varphi(y) e^{x} \Gamma(y) V+C
\end{aligned}
$$

or

$$
\begin{aligned}
f(x, y)= & 6 \varphi(y) e^{x} A+e^{x} B+\left(6 \varepsilon \varphi(y) e^{x} \Lambda(y)-\frac{e^{3 x}}{24 c_{1}}\right) T \\
& +\frac{1}{c}\left(1-\frac{\varphi(y)}{\varphi(0)}\right) e^{x} V+C,
\end{aligned}
$$


where $A, B, T, V$, and $C$ are constant vectors. The second equation (6.2) occurs if $k=0$. In both cases, the constant principal curvature $\lambda$ is zero.

Proof. The following are straightforward consequences of $\sigma=-2 a$, and equations $(2.8-2.9)$

$$
Y a=-a b
$$

We also get an immediate simplification of (2.7) to $\lambda=0$, and hence $\mu=c e^{-x}$. Also, the second Gauss equation (2.6), simplifies to become

$$
-2 X a+2 \varepsilon Y b+6 \varepsilon b^{2}+6 a^{2}=-\varepsilon \mu .
$$

The equation for $\tau$ in this case reduces to $(X \tau) / \tau=-a$. Since $(X b) / b=a$ by (6.4), we may set

$$
\tau=\frac{1}{b}
$$

so

$$
X=-2 a \frac{\partial}{\partial x}, \quad Y=b \frac{\partial}{\partial y} .
$$

Since $a$ and $b$ are nonzero, equations (6.3) and (6.4) become

$$
a_{y}=-a, \quad-2 b_{x}=b,
$$

which allows us to solve for $a$ and $b$, to get

$$
a=l(x) e^{-y}, \quad b=p(y) e^{-x / 2}
$$

for some functions $l$ and $p$. Using this information, the second Gauss equation (6.5) can be rewritten as

$$
4 l l_{x} e^{-2 y}+2 \varepsilon p p_{y} e^{-x}+6 \varepsilon p^{2} e^{-x}+6 l^{2} e^{-2 y}=-\varepsilon c e^{-x},
$$

which can be reformulated as

$$
\left(4 l l_{x}+6 l^{2}\right) e^{-2 y}=-\varepsilon\left(2 p p_{y}+6 p^{2}+c\right) e^{-x} .
$$

Since both sides of this equation are products of a function of $x$ and a function of $y$, there must be a constant value $k$ such that

$$
\begin{gathered}
4 l l_{x}+6 l^{2}=k e^{-x}, \\
2 p p_{y}+6 p^{2}+c=-\varepsilon k e^{-2 y} .
\end{gathered}
$$

These two ordinary differential equations can be solved to get

$$
l(x)^{2}=\frac{1}{4} k e^{-x}+c_{1} e^{-3 x},
$$




$$
p(y)^{2}=-\frac{c}{6}-\frac{k \varepsilon}{4} e^{-2 y}+c_{2} e^{-6 y},
$$

where $c_{1}$ and $c_{2}$ are constants. These functions determine $a$ and $b$ up to a sign, and we can then calculate that

$$
\begin{gathered}
\frac{a_{x}}{a}=\frac{l_{x}}{l}=\frac{k}{k+4 c_{1} e^{-2 x}}-\frac{3}{2}, \quad \frac{\varepsilon b^{2}}{2 a^{2}}=\frac{\varepsilon}{2} \frac{p^{2} e^{2 y}}{l^{2} e^{x}}, \\
\frac{b_{y}}{b}=\frac{p_{y}}{p} .
\end{gathered}
$$

Now, using the fact that $f=e^{x} g(y)+h(x)$, and $\xi=-c g(y)+V$, from (4.6) and (4.7), we can rewrite (4.3) and (4.4) to get

$$
\begin{aligned}
& e^{x} g(y)+h_{x x}=-\left(\frac{1}{2}+\frac{a_{x}}{a}\right)\left(e^{x} g(y)+h_{x}\right) \\
&-\frac{\varepsilon}{2} \frac{p^{2} e^{2 y}}{l^{2}} g_{y}-\frac{c \varepsilon e^{2 y}}{4 l^{2}} g(y)+\frac{\varepsilon e^{2 y}}{4 l^{2}} V, \\
& e^{x} g_{y y}=\left(1-\frac{p_{y}}{p}\right) e^{x} g_{y}-c \frac{e^{x}}{p^{2}} g(y)+\frac{e^{x}}{p^{2}} V .
\end{aligned}
$$

Now, we can rewrite (6.10) as follows:

$$
\left(\frac{3}{2}+\frac{a_{x}}{a}\right) e^{x} g(y)+\frac{c \varepsilon e^{2 y}}{4 l^{2}} g(y)+\frac{2 \varepsilon p^{2} e^{2 y}}{4 l^{2}} g_{y}-\frac{\varepsilon e^{2 y}}{4 l^{2}} V=-h_{x x}-\left(\frac{1}{2}+\frac{a_{x}}{a}\right) h_{x},
$$

and by applying the simplifications given in (6.8) and (6.9), this can be rewritten

$$
\left(k+c \varepsilon e^{2 y}\right) g(y)+2 \varepsilon p^{2} e^{2 y} g_{y}-\varepsilon e^{2 y} V=-4 l^{2} h_{x x}+4 c_{1} e^{-3 x} h_{x} .
$$

Notice that the left side of this equation is a function of $y$ while the right side is a function of $x$. Therefore both sides must be a constant vector, say $T$; i.e.,

$$
\begin{gathered}
\left(k+c \varepsilon e^{2 y}\right) g(y)+2 \varepsilon p^{2} e^{2 y} g_{y}-\varepsilon e^{2 y} V=T, \\
-4 l^{2} h_{x x}+4 c_{1} e^{-3 x} h_{x}=T .
\end{gathered}
$$

Equation (6.12) may be solved by a straightforward but onerous calculation which allows us to conclude that there is a constant vector $A$ such that

$$
g(y)=6 \sqrt{12 c_{2}-3 \varepsilon k e^{4 y}-2 c e^{6 y}}\left(\int_{0}^{y} \frac{e^{6 t} V+\varepsilon e^{4 t} T}{\left(12 c_{2}-3 \varepsilon k e^{4 t}-2 c e^{6 t}\right)^{3 / 2}} d t+A\right) .
$$

Now (6.13) can be rewritten as

$$
\frac{d}{d x}\left(e^{-x}\left(4 c_{1}+k e^{2 x}\right)^{1 / 2} h_{x}\right)=-e^{2 x}\left(4 c_{1}+k e^{2 x}\right)^{-1 / 2} T .
$$


Solutions of (6.13) take two different forms depending on $k$. Note that we must have $k+4 c_{1}>0$ since the solution must be defined at $(0,0)$.

If $k \neq 0$, then the solutions of (6.14) are given by

$$
h(x)=\left(\int_{0}^{x} \frac{e^{t}}{\left(4 c_{1}+k e^{2 t}\right)^{1 / 2}} d t\right) B-\frac{e^{x}}{k} T+C,
$$

where again $B$ and $C$ are constant vectors.

Now, let

$$
\begin{gathered}
\varphi(y)=\left(12 c_{2}-3 \varepsilon k e^{4 y}-2 c e^{6 y}\right)^{1 / 2} \\
\Gamma(y)=\int_{0}^{y} \frac{e^{6 t}}{\varphi(t)^{3}} d t, \quad \Lambda(y)=\int_{0}^{y} \frac{e^{4 t}}{\varphi(t)^{3}} d t \\
\Psi(x)=\int_{0}^{x} \frac{e^{t}}{\left(4 c_{1}+k e^{2 t}\right)^{1 / 2}} d t
\end{gathered}
$$

Then

$$
\begin{gathered}
g(y)=6 \varphi(y)(A+\Gamma(y) V+\varepsilon \Lambda(y) T), \\
h(x)=\Psi(x) B-\frac{e^{x}}{k} T+C .
\end{gathered}
$$

Then, by (4.6), we can write the immersing function as

$$
\begin{aligned}
f(x, y)= & 6 \varphi(y) e^{x} A+\Psi(x) B+\left(6 \varepsilon \varphi(y) e^{x} \Lambda(y)-\frac{e^{x}}{k}\right) T \\
& +6 \varphi(y) e^{x} \Gamma(y) V+C
\end{aligned}
$$

We now look at the case $k=0$. The function $g(y)$ still takes the form given in (6.19), but $c_{1}$ must be positive and the solution to (6.14) is

$$
h(x)=-\frac{e^{3 x}}{24 c_{1}} T+e^{x} B+C,
$$

where $B$ and $C$ are constant vectors. Since $\varphi$ simplifies somewhat, the integral for $\Gamma(y)$ in $(6.17)$ can be expressed as

$$
\Gamma(y)=\frac{1}{6 c}\left(\frac{1}{\varphi(y)}-\frac{1}{\varphi(0)}\right) .
$$

Then we can write

$$
\begin{aligned}
f(x, y)= & 6 \varphi(y) e^{x} A+e^{x} B+\left(6 \varepsilon \varphi(y) e^{x} \Lambda(y)-\frac{e^{3 x}}{24 c_{1}}\right) T \\
& +\frac{1}{c}\left(1-\frac{\varphi(y)}{\alpha}\right) e^{x} V+C .
\end{aligned}
$$


Remark. Clearly, the integral in (6.15) can be evaluated explicitly. It does not seem worthwhile to do so since there are other integrals in this section for which we cannot do the same. We point out, however, that complete evaluation has been done for a similar integral in Case 1.

The solutions just obtained involve certain real and vector constants. Although there are many degrees of freedom, the constants are not entirely arbitrary. We look at the implications of the condition $\nu=\theta$ beginning with $k \neq 0$. First note that

$$
\begin{aligned}
& f_{x}= 6 \varphi(y) e^{x} A+\frac{e^{x}}{\left(4 c_{1}+k e^{2 x}\right)^{1 / 2}} B+\left(6 \varepsilon \varphi(y) e^{x} \Lambda(y)-\frac{e^{x}}{k}\right) T \\
&+6 \varphi(y) e^{x} \Gamma(y) V \\
& f_{y}=6 \varphi_{y} e^{x} A+\left(6 \varepsilon \varphi_{y} e^{x} \Lambda(y)+6 \varepsilon \varphi(y) e^{x} \Lambda_{y}\right) T \\
&+\left(6 \varphi_{y} e^{x} \Gamma(y)+6 \varphi(y) e^{x} \Gamma_{y}\right) V \\
& \xi=-6 \varphi(y) c A+(1-6 \varphi(y) c \Gamma(y)) V-6 \varepsilon \varphi(y) c \Lambda(y) T
\end{aligned}
$$

In view of (4.1) in which $X$ and $Y$ are orthonormal with respect to the affine metric $h$, we have

$$
\nu\left(f_{x}, f_{y}\right)= \pm \frac{\tau}{\sigma}= \pm \frac{1}{2 a b}=\frac{2 \sqrt{3} e^{2 x+4 y}}{\left(4 c_{1}+k e^{2 x}\right)^{1 / 2}\left(12 c_{2}-3 \varepsilon k e^{4 y}-2 c e^{6 y}\right)^{1 / 2}} .
$$

In order to minimize calculation, we evaluate $\theta\left(f_{x}, f_{y}\right)$ at $(x, 0)$ first. It turns out that if the term in (6.20) involving $B$ is omitted, the result is 0 . Thus a necessary condition is that $B$ is nonzero. Now suppose that $\operatorname{det}(A, T, V) \neq 0$. Write $B=b_{1} A+b_{2} T+b_{3} V$. Then one can verify that

$$
\theta\left(f_{x}, f_{y}\right)=\frac{6 \varepsilon e^{2 x}}{\alpha^{2}\left(4 c_{1}+k e^{2 x}\right)^{1 / 2}}\left(b_{1}+6 \alpha\left(k b_{2}+c b_{3}\right)\right) \operatorname{det}(A, T, V)
$$

where $\alpha=\varphi(0)$. Setting $y=0$ in (6.23) and comparing with $\theta\left(f_{x}, f_{y}\right)$, we see that

$$
\operatorname{det}(A, T, V)=\frac{\alpha \varepsilon}{\sqrt{3}\left(b_{1}+6 \alpha\left(k b_{2}+c b_{3}\right)\right.} .
$$

This can be regarded as a restriction on $B$, or on $A, T$, and $V$. By applying an appropriate affine transformation to the original surface, we can arrange that $A, T$, and $V$ are the standard unit basis vectors for $\mathbb{R}^{3}$ and thus the condition reduces to

$$
\alpha \varepsilon=\sqrt{3}\left(b_{1}+6 \alpha\left(k b_{2}+c b_{3}\right)\right) .
$$

On the other hand, it could be that $\operatorname{det}(V, A, T)=0$ but that any two of these vectors are linearly independent. We can again find a sufficient condition relating the four constant vectors. For example, if $V=r A+s T$, then we must have

$$
\operatorname{det}(B, T, A)=\frac{\alpha \varepsilon}{\sqrt{3}(6 k \alpha s-6 \alpha c+r)} .
$$


Again, by a suitable affine transformation, we can arrange that the determinant in this equation is 1 . Similar conditions can be derived for the other possibilities of this type.

Finally, it might be that two of the vectors are proportional. For example, if $A=r V$, we need

$$
\operatorname{det}(B, T, V)=\frac{\alpha \varepsilon}{\sqrt{3}(1-6 \alpha c r)} .
$$

This puts a restriction on $r$, namely $6 \alpha c r \neq 1$, and when $r$ is chosen, it puts a further restriction on $B, T$, and $V$. Note that $A=0$ is included in the possibilities here.

For the solutions with $k=0$, we compute

$$
\begin{gathered}
f_{x}(x, 0)=6 \alpha e^{x} A-\frac{e^{3 x}}{8 c_{1}} T+e^{x} B, \\
f_{y}(x, 0)=6 \varphi_{y} e^{x} A+6 \varepsilon \alpha e^{x} \Lambda_{y} T-\frac{1}{c \alpha} \varphi_{y} e^{x} V .
\end{gathered}
$$

But differentiating (6.16) gives $\varphi_{y}(0)=-\frac{6 c}{\alpha}$ when $k=0$. Further, from (6.17), $\Lambda_{y}(0)=\frac{1}{\alpha^{3}}$. Thus, we can write

$$
\begin{gathered}
f_{y}(x, 0)=-\frac{36 c e^{x}}{\alpha} A+\frac{6 \varepsilon e^{x}}{\alpha^{2}} T+\frac{6 e^{x}}{\alpha^{2}} V, \\
\xi(x, 0)=-c g(0)+V=-6 c \alpha A+V, \\
\theta\left(f_{x}, f_{y}\right)=\frac{36 \varepsilon e^{2 x}}{\alpha} \operatorname{det}(A, T, V)+\frac{6 \varepsilon e^{2 x}}{\alpha^{2}} \operatorname{det}(B, T,-6 c \alpha A+V),
\end{gathered}
$$

while

$$
\nu\left(f_{x}, f_{y}\right)=\frac{\sqrt{3} e^{2 x}}{\sqrt{c_{1}} \alpha} .
$$

If $\operatorname{det}(A, T, V) \neq 0$, we can express $B=b_{1} A+b_{2} T+b_{3} V$ and obtain the relationship

$$
\operatorname{det}(A, T, V)=\frac{\alpha \varepsilon}{2 \sqrt{3} \sqrt{c_{1}}\left(6 \alpha+b_{1}+6 c \alpha b_{3}\right)} .
$$

On the other hand, if $\operatorname{det}(A, T, V)=0$, some three of the vectors $B, A, T$, and $V$ must be linearly independent. This gives some condition analogous to that obtained for $k \neq 0$.

7. CASE $3: b=0$ AND $2 a+\sigma \neq 0 . \nabla_{Y} Y$ IS A MUltiple of $X$

Theorem 6. Let $f: M \rightarrow \mathbb{R}^{3}$ be an affine Dupin surface with exactly one nonconstant principal curvature $\mu$ and planar lines of curvature. Let $X$ and $Y$ be orthonormal (with respect to $h$ ) principal vectors corresponding to principal curvatures $\lambda$ and $\mu$ respectively. Suppose that $\nabla_{Y} Y$ is a nonzero multiple of $X$. Let $p_{0}$ be a point of $M$ where $d \mu \neq 0$. Then there is a coordinate system $\{x, y\}$ with origin $p_{0}$ such that in the domain of these coordinates,

$$
f(x, y)=e^{x} g(y)+h(x)
$$


where

$$
\begin{gathered}
g(y)=\frac{y^{2}}{2} K+y A+B, \\
h(x)=e^{x} \mu\left(\int_{0}^{x} \frac{d t}{e^{2 t} \mu(t)^{2} \sigma(t)^{2 / 3}}\right) K-\frac{e^{x}}{c} V+e^{x} \mu C,
\end{gathered}
$$

or $g(y)$ is one of

$$
\begin{gathered}
\cosh (\omega y) A+\sinh (\omega y) B-\frac{K}{\omega^{2}} \\
\cos (\omega y) A+\sin (\omega y) B+\frac{K}{\omega^{2}}
\end{gathered}
$$

and

$$
h(x)=\frac{\varepsilon(2 a+\sigma) \tau^{3}}{e^{2 x}}\left(\left(\int_{0}^{x} \frac{e^{2 t} d t}{\sigma(2 a+\sigma)^{2} \tau^{3}}\right) V-\left(\int_{0}^{x} \frac{e^{3 t} d t}{\sigma(2 a+\sigma)^{2} \tau^{5}}\right) K+\frac{\varepsilon}{\alpha} C\right),
$$

where $\lambda, c \neq 0$, and $\omega>0$ are real constants, $A, B, K, V$, and $C$ are constant vectors, $a$ and $\sigma$ are functions of $x$ determined up to constants by certain differential equations (see (7.1) and (7.2)). Also, $\alpha$ denotes the value of $(2 a+\sigma) \sigma$ at $x=0$, $\mu=\lambda+c e^{-x}$, and $\tau=\omega(\mu+\varepsilon \sigma(2 a+\sigma))^{-\frac{1}{2}}$.

Proof. We assume the conditions of Theorem 3 and $b=0$. Since $\lambda$ is constant, we have $\gamma=0$. Then from (2.8) and (2.3), we see that

$$
Y a=0, \quad Y \sigma=0 .
$$

The second and third Gauss equations become

$$
\begin{gathered}
X \sigma-a \sigma+\sigma^{2}=-\varepsilon \mu, \\
\varepsilon X(2 a+\sigma)+6 \varepsilon a^{2}+5 \varepsilon a \sigma+\varepsilon \sigma^{2}=-\lambda .
\end{gathered}
$$

In local coordinates,

$$
\begin{gathered}
\sigma \sigma_{x}-a \sigma+\sigma^{2}=-\varepsilon\left(\lambda+c e^{-x}\right), \\
2 \varepsilon \sigma a_{x}+\varepsilon \sigma \sigma_{x}+6 \varepsilon a^{2}+5 \varepsilon a \sigma+\varepsilon \sigma^{2}=-\lambda .
\end{gathered}
$$

It is not immediately apparent what the function $\tau$ should be. It turns out that $\tau=e^{x} \sigma^{1 / 3}$ is satisfactory, see (7.10) below. Meanwhile, we note that $X(\ln \tau)=a+\sigma$ has solutions with no dependency on $y$ so that we may assume $\tau_{y}=0$ in (4.4).

Now (4.3) may be rewritten as

$$
\begin{aligned}
e^{x} g(y)+h_{x x}= & \left(\frac{a-\sigma_{x}}{\sigma}\right)\left(e^{x} g(y)+h_{x}\right)-\frac{\varepsilon \lambda e^{x}}{\sigma^{2}} g(y) \\
& -\frac{\varepsilon c}{\sigma^{2}} g(y)-\frac{\varepsilon \lambda}{\sigma^{2}} h(x)+\frac{\varepsilon}{\sigma^{2}} V
\end{aligned}
$$


The terms involving $g(y)$ drop out because of (7.1), and thus (7.31) simplifies to

$$
h_{x x}=\left(\frac{a-\sigma_{x}}{\sigma}\right) h_{x}-\frac{\varepsilon \lambda}{\sigma^{2}} h(x)+\frac{\varepsilon}{\sigma^{2}} V .
$$

Further, (4.4) can be written as

$$
e^{x} g_{y y}=-\varepsilon \tau^{2} \sigma(2 a+\sigma)\left(e^{x} g(y)+h_{x}\right)-\tau^{2}\left(\left(\lambda e^{x}+c\right) g(y)+\lambda h(x)\right)+\tau^{2} V,
$$

or

$$
\begin{aligned}
g_{y y}+ & \left(\varepsilon \tau^{2} \sigma(2 a+\sigma)+\tau^{2} \mu\right) g(y) \\
& =-\varepsilon \tau^{2} \sigma(2 a+\sigma) e^{-x} h_{x}-\tau^{2} \lambda e^{-x} h(x)+\tau^{2} e^{-x} V .
\end{aligned}
$$

We would now like to solve for $g(y)$. Examining (7.4) shows that we can rewrite this equation as

$$
g_{y y}-t(x) g(y)=L(x)
$$

where $t(x)$ and $L(x)$ are real- and vector-valued functions respectively.

At this point, various solutions are possible. We will first consider the case that $t(x)$ is identically zero. Since the left side of (7.5) is a function of $y$ alone while the right side is function of $x$, there is a constant vector $K$ such that

$$
g_{y y}=L(x)=K .
$$

Since $\tau \neq 0, t(x)=0$ reduces to $\varepsilon \sigma(2 a+\sigma)+\mu=0$ and hence

$$
a=-\frac{\sigma^{2}+\varepsilon \mu}{2 \sigma} .
$$

Then (7.1) becomes

$$
2 \sigma \sigma_{x}+3 \sigma^{2}+3 \varepsilon \mu=0
$$

This can be solved to get

$$
\sigma^{2}=c_{1} e^{-3 x}-\varepsilon \lambda-\frac{3}{2} \varepsilon c e^{-x}
$$

Consequently, we know that

$$
a^{2}=\frac{\left(\frac{3}{2} \varepsilon c e^{-x}-3 c_{1} e^{-3 x}\right)^{2}}{36 \sigma^{2}} .
$$

$X(\ln \tau)=a+\sigma$ leads to

$$
\frac{\tau_{x}}{\tau}=1+\frac{1}{3} \frac{\sigma_{x}}{\sigma}
$$

which has $\tau=e^{x} \sigma^{1 / 3}$ as a solution. In view of Lemma 4.3, we may assume that our coordinate system uses this particular $\tau$. Equating the right side of (7.4) to $K$ yields

$$
\frac{e^{x}}{\tau^{2}} K=V-\lambda h(x)+\mu h_{x}
$$


which can be rewritten as

$$
\left(\frac{e^{-x}}{\mu} h\right)_{x}=\frac{1}{e^{2 x} \mu^{2} \sigma^{2 / 3}} K-\frac{e^{-x}}{\mu^{2}} V
$$

Solving for $h$, we find that there is a constant vector $C$ such that

$$
h(x)=e^{x} \mu\left(\int_{0}^{x} \frac{d t}{e^{2 t} \mu(t)^{2} \sigma(t)^{2 / 3}}\right) K-\frac{e^{x}}{c} V+e^{x} \mu C,
$$

while the solution of (7.5) is given by

$$
g(y)=\frac{y^{2}}{2} K+y A+B
$$

for some constant vectors $A$ and $B$. Now, if we let

$$
\Psi(x)=\int_{0}^{x} \frac{1}{e^{2 t} \mu(t)^{2} \sigma(t)^{2 / 3}} d t
$$

we can write

$$
\begin{aligned}
f(x, y) & =y e^{x} A+e^{x} B+e^{x} \mu C+e^{x}\left(\mu \Psi(x)+\frac{y^{2}}{2}\right) K-\frac{e^{x}}{c} V \\
& =y e^{x} A+e^{x}\left(B+\lambda C-\frac{1}{c} V\right)+e^{x}\left(\mu \Psi(x)+\frac{y^{2}}{2}\right) K+c C .
\end{aligned}
$$

Note that $A, B+\lambda C-\frac{1}{c} V$, and $K$ must be linearly independent. Then a suitable affine transformation applied to the original surface brings it to the surface

$$
f(x, y)=\left[\left(y e^{x}, e^{x}, e^{x}\left(\mu \Psi(x)+\frac{y^{2}}{2}\right)\right],\right.
$$

whose affine normal is given by

$$
\xi=\left[-e^{x} \mu y,-e^{x} \mu,-e^{x} \mu\left(\frac{y^{2}}{2}+\lambda \Psi(x)\right)\right] .
$$

The condition $\theta=\nu$ will be automatically satisfied (modulo orientation). In fact, direct computation gives

$$
\theta\left(f_{x}, f_{y}\right)=-\frac{e^{x}}{\sigma^{2 / 3}}
$$

and

$$
\nu\left(f_{x}, f_{y}\right)= \pm \frac{e^{x}}{\sigma^{2 / 3}}
$$

We now consider the case that $t(x) \neq 0$. In this case, (7.5) has as solution

$$
g(y)=\cosh (\omega y) A+\sinh (\omega y) B-\frac{L(x)}{t(x)}
$$


or

$$
g(y)=\cos (\omega y) A+\sin (\omega y) B-\frac{L(x)}{t(x)}
$$

where $A$ and $B$ are vector-valued functions of $x$ and $\omega$ is a positive function of $x$ satisfying $\omega^{2}= \pm t(x)$. But noting that $g$ depends only on $y$, and considering the values of $g^{\prime}(0), g^{\prime \prime}(0)$, etc. one can check that $\omega^{2}$ is constant and hence that both $t(x)$ and $L(x)$ must be constant, say $L(x)=K$, and $t(x)=k$. Then $A, B$ and $\omega$ are constant as well and, from equation (7.4), we see that

$$
\begin{gathered}
\varepsilon \tau^{2} \sigma(2 a+\sigma)+\tau^{2} \mu=k, \\
-\varepsilon \tau^{2} \sigma(2 a+\sigma) e^{-x} h_{x}-\tau^{2} \lambda e^{-x} h(x)+\tau^{2} e^{-x} V=K .
\end{gathered}
$$

Therefore if we let $\eta=\sigma(2 a+\sigma)$, then (7.10) shows that

$$
\tau^{2}=\frac{k}{\varepsilon \eta+\mu}
$$

and so (7.11) can be rewritten,

$$
\varepsilon \eta h_{x}+\lambda h=V-\frac{(\varepsilon \eta+\mu) e^{x}}{k} K
$$

As an integrating factor for this equation, we need to compute the integral $\int \frac{1}{\eta} d x$. Dividing both sides of $(7.2)$ by $\sigma(2 a+\sigma)$ gives

$$
-\frac{\varepsilon \lambda}{\sigma(2 a+\sigma)}=\frac{d}{d x} \ln (2 a+\sigma)+\frac{3 a}{\sigma}+1 .
$$

Since $\frac{a}{\sigma}=\frac{d}{d x} \ln (\tau)-1$, we have

$$
\frac{\varepsilon \lambda}{\sigma(2 a+\sigma)}=-\frac{d}{d x} \ln \left((2 a+\sigma) \tau^{3}\right)+2
$$

Let

$$
G(x)=2 x-\ln \left((2 a+\sigma) \tau^{3}\right)+\ln \alpha
$$

where $\alpha$ is the value of $(2 a+\sigma) \tau^{3}$ at $x=0$. This is to make $G(0)=0$. We rewrite (7.12) as

$$
\left(e^{G(x)} h\right)_{x}=\frac{e^{G(x)}}{\varepsilon \eta} V-\frac{e^{G(x)} e^{x}}{k}\left(1+\frac{\mu}{\varepsilon \eta}\right) K
$$

Letting

$$
v(x)=\varepsilon \int_{0}^{x} \frac{e^{G(t)}}{\eta(t)} d t, \quad w(x)=\frac{1}{k} \int_{0}^{x} e^{G(t)+t}\left(1+\frac{\varepsilon \mu(t)}{\eta(t)}\right) d t,
$$

we can now write down the solution of (7.12) as

$$
h(x)=v(x) e^{-G(x)} V-w(x) e^{-G(x)} K+e^{-G(x)} C
$$

where $C$ is a constant vector. Alternatively, using the fact that

$$
e^{G(x)}=\frac{\alpha e^{2 x}}{(2 a+\sigma) \tau^{3}}
$$

we can express $h(x)$ in terms of $a, \sigma$, and $\tau$ as follows:

$$
h(x)=\frac{\varepsilon(2 a+\sigma) \tau^{3}}{e^{2 x}}\left(\left(\int_{0}^{x} \frac{e^{2 t} d t}{\sigma(2 a+\sigma)^{2} \tau^{3}}\right) V-\left(\int_{0}^{x} \frac{e^{3 t} d t}{\sigma(2 a+\sigma)^{2} \tau^{5}}\right) K+\frac{\varepsilon}{\alpha} C\right) .
$$

Verifying that $\nu=\theta$ in this case is a tedious exercise. 


\section{THE LOCAL CLASSIFICATION}

We now prove the main theorem.

Theorem 7. Let $f: M \rightarrow \mathbb{R}^{3}$ be an affine Dupin surface with (distinct real) principal curvatures $\lambda$ and $\mu$ and planar lines of curvature. Then there is a dense set of points of $M$, each of which is of one of the types $0-3$.

Proof. Let $p$ be an arbitrary point of $M$. Suppose that every neighborhood $U$ of $p$ contains a point $p_{U}$ where $d \mu \neq 0$. Then, either $p_{U}$ is a type 1 point, or there are points satisfying Case 2 or Case 3 as close to $p_{U}$ as we like. In either case, $U$ contains a point of one of the types $1-3$. The other possibility is that $p$ has a neighborhood where $d \lambda=d \mu=0$, so that $p$ itself is of type 0 .

Thus the set of points of types $0-3$ is dense in $M$.

\section{Concluding REMARKS}

In this paper, we have introduced a wide class of affine surfaces that have not been studied before. The simplest (Case 1) are the first explicit examples with one constant and one nonconstant principal curvature. It will be interesting to examine particular surfaces arising from Case 2 and Case 3 more closely in the future. One such example (Case 2) is $\left(W+V^{3}\right)^{3} U^{2}=V^{5}$ (see [NR2] for other examples).

The question of the necessity of assuming that the lines of curvature are planar remains open and is also an interesting subject for future study.

Finally, there is the question of improving Theorem 7. We know of no example having points that are not of types $0-3$. Perhaps a more comprehensive analysis of the results of Theorems 4-6 will show that any surface satisfying the hypothesis of Theorem 3 must have all its points of the same type. This is also a good question for future study.

\section{REFERENCES}

[C] T. E. Cecil, Lie sphere geometry with applications to submanifolds, Universitext, SpringerVerlag, New York, Berlin, Heidelberg, 1992. MR 94m:53076

[CR] T. E. Cecil and P. J. Ryan, Tight and taut immersions of manifolds, Research Notes in Math., vol. 107, Pitman, Boston, London, Melbourne, 1985. MR 87b:53089

[K] W. Klingenberg, A course in differential geometry, Graduate Texts in Math., vol. 51, Springer-Verlag, New York, Heidelberg, Berlin, 1978. MR 57:13702

[MR] M. A. Magid and P. J. Ryan, Flat affine spheres in $\mathbf{R}^{3}$, Geom. Dedicata 33 (1990), 277288. MR 91e:53016

[NR1] R. Niebergall and P. J. Ryan, Affine isoparametric hypersurfaces, Math. Z. 217 (1994), 479-485. MR 95:05

[NR2] _ Focal sets in affine geometry, Geometry and Topology of Submanifolds VI, World Scientific, 1994. MR 95:07

[N] K. Nomizu, Introduction to affine differential geometry, Part I, MPI/88-37, Bonn (1988).

[NS] K. Nomizu and T. Sasaki, A new model of unimodular-affinely homogeneous surface, Manuscripta Math. 73 (1991), 39-44. MR 92h:53014

[V] L. Vrancken, Affine surfaces with constant affine curvatures, Geom. Dedicata 33 (1990), 177-194. MR 91k:53022

Department of Mathematics and Computer Science, University of Northern British Columbia, Prince George, BC, Canada V2L 5P2

E-mail address: rossn@unbc.edu

Department of Mathematics and Statistics, McMaster University, Hamilton, OnTARIO, CANADA, L8S $4 \mathrm{~K} 1$

E-mail address: pjr@maccs.dcss.mcmaster.ca 